\title{
Compound self-motion perception induced by two kinds of optical motion
}

\author{
HIROYUKI ITO and CHISA FUJIMOTO \\ Kyushu Institute of Design, Fukuoka, Japan
}

\begin{abstract}
Two kinds of flow patterns consisting of random dots were presented simultaneously to subjects to investigate whether or not two kinds of vection occur simultaneously. One pattern induces vertical linear self-translation, whereas the other induces self-rotation around a vertical axis (when either pattern is presented alone). Three sets of conditions were tested. The first condition was one in which random dots moved in a summed direction of both flow vectors. In the second condition, both flow patterns were simply overlaid, whereas in the third condition, the two kinds of flow patterns were overlaid with a depth separation produced by binocular disparity. The subjects perceived both kinds of vection simultaneously in directions opposite to those of the corresponding flow components under the first condition, whereas either vection occurred mainly under the second condition. Under the third condition, both of the flows induced each kind of vection simultaneously, despite there being no physical vector summation of dot motion. The background flow induced vection in a direction opposite to the flow direction, whereas the foreground flow induced vection in the same direction as the flow direction. These results show that induced self-translation and induced self-rotation can occur simultaneously in two ways.
\end{abstract}

When one observes optical motion covering a large region in the visual field, self-motion in the direction opposite to that of the visual motion is sometimes induced. This phenomenon has been called vection and is classified as being of two types: induced self-translation and induced self-rotation.

In a laboratory setting, induced self-translation has been investigated with the motion of linear elements on a flat screen (Johansson, 1977; Kano, 1991; Nakamura \& Shimojo, 1998, 1999, 2000) or with expanding flow patterns that induce forward self-translation (Andersen \& Braunstein, 1985; Ohmi \& Howard, 1988; Palmisano, 1996; Palmisano, Gillam, \& Blackburn, 2000). Induced vertical self-translation seems to occur sooner and for longer durations than that in horizontal directions - that is, along the left-right axis or the visual axis-when an observer is in an upright position (Kano, 1991; Nakamura \& Shimojo, 1998).

On the other hand, typically, when one sits in a large rotating textured cylinder, induced self-rotation in the direction opposite to that of the cylinder's rotation occurs (Brandt, Dichgans, \& Koenig, 1973; Howard \& Heckmann, 1989). Traditionally, a vertical cylinder has been used to investigate the characteristics of induced self-

We thank Tadahide Shibao and Asako Takenaka for their assistance in making the apparatus and collecting the data. We also thank Shinji Nakamura, Shoji Sunaga, and Noriyuki Kitajima for their helpful comments. Correspondence concerning this article should be addressed to H. Ito, Department of Visual Communication Design, Kyushu Institute of Design, 4-9-1, Shiobaru, Minami-ku, Fukuoka-shi, 815-8540 Japan (e-mail: ito@kyushu-id.ac.jp). rotation (see Ito \& Shibao, 1999). However, when a large sphere is used, the rotation axis itself can be a variable. Perception of body rotation or tilt around a vertical axis (yaw) seems to occur more strongly than that around a horizontal axis - that is, an axis passing through both ears (pitch) or the visual axis (roll; Howard, Cheung, \& Landolt, 1988).

Usually, these kinds of vections are tested separately. However, it is obvious that humans experience more complicated self-motion. One of our primary objectives in the present research was to examine the occurrence of compound self-motion perception from optical motion, which has never been reported. We chose vertical selftranslation and self-rotation around a vertical axis, both of which are relatively easy to produce in a laboratory, as was noted above, and tested to see whether or not their compound vection occurs, using their vector-summed flows. Whether these two types of vections are mutually inhibitory or able to coexist is an open question.

The second objective was to investigate the effect on vection of superimposing two types of flow patterns. Ohmi, Howard, and Landolt (1987) reported that when two flows existed, the farther one determined the vection direction. Ohmi et al. and Ohmi and Howard (1988) also showed that when a stationary object was seen at a farther position, vection was inhibited. This depth order effect does not require a stereo process. When two flow patterns moving in opposite directions existed on the same plane, apparent reversals of the depth order reversed the direction of vection. In the present experiment, we overlaid two flow patterns on a screen, each of which alone would induce a sense of self-rotation or self- 
translation, with no cues as to their depth order. If only the perceptually farther flow induces vection, which vection would be perceived would vary according to the apparent reversal of the depth order, as was observed by Ohmi et al. On the other hand, if global vector summation occurs in the visual system or if both flows are processed in parallel, the perceived vection would be the same as that under the vector-summed flow condition noted above.

The effect of depth separation produced by binocular stereopsis on the coexistence of the two kinds of vections was also tested here. Although the background flow determines vection direction, as was noted above, contributions of foreground-plane motion have also been pointed out (Howard \& Heckmann, 1989; Nakamura \& Shimojo, 1999, 2000). Howard and Heckmann found that when horizontal dot motion was displayed on a foreground screen against a farther peripheral background on which stationary dots were displayed, a small amount of vection in the same direction as the dot motion on the foreground plane occurred. Nakamura and Shimojo (1999) demonstrated nonlinear interactions between foreground- and background-plane motions for vection, which translate in the same or opposite directions with respect to each other. Nakamura and Shimojo (2000) also showed vection in the same direction as that of the foreground flow when an orthogonal flow was presented in the background. We also present two flows separated in stereoscopic depth, each of which alone would induce a different kind of vection, a sense of self-translation or of self-rotation. If the flow at a perceptually farther position dominates in the induction of vection, the stereoscopic depth order of the two types of flows might determine which types of vection would occur, self-translation or self-rotation. However, if the perceptually closer flow also has an effect on the induction of vection, as was reported by Nakamura and Shimojo (2000), it is possible that the two types of vection would coexist.

\section{METHOD}

\section{Subjects}

Eleven subjects, including the authors, participated in the experiment under the linear flow, circular flow, vector sum, and overlay conditions, noted below. All the subjects had normal or correctedto-normal vision, and all were naive volunteers, with the exception of the authors. Under linear-near and circular-near conditions, 10 subjects, including 4 of the subjects above, participated in the experiment. All the subjects had normal or corrected-to-normal vision with contact lenses. Glasses were not permitted here because the subjects were required to wear anaglyph glasses under the linearnear and circular-near conditions, as will be noted below. All but 1 (the second author) were naive volunteers.

\section{Apparatus and Stimuli}

As is shown in Figure 1, a darkened room was made using metal frames covered with black cloth. Three translucent screens, similar to windows, surrounded the subject. The size of each screen was $80 \mathrm{~cm}$ (horizontal) $\times 60 \mathrm{~cm}$ (vertical). The other sections of the wall were covered with black cloth. Viewing distance was $69.3 \mathrm{~cm}$. The visual angle of the screens was $180^{\circ}$ (horizontally) and $46.4^{\circ}$ (vertically). The wide angles, especially in the horizontal dimension, contributed to the rapid and strong induction of vection. On each screen, moving random-dot patterns were projected from behind. The stimulus patterns were generated by a computer (Sharp CZ-644C) and were projected by three NTSC LCD projectors (Sharp XV-P3, having an LCD panel consisting of 301,158 pixels). Although the resolution was not so high, the quality of motion dis-

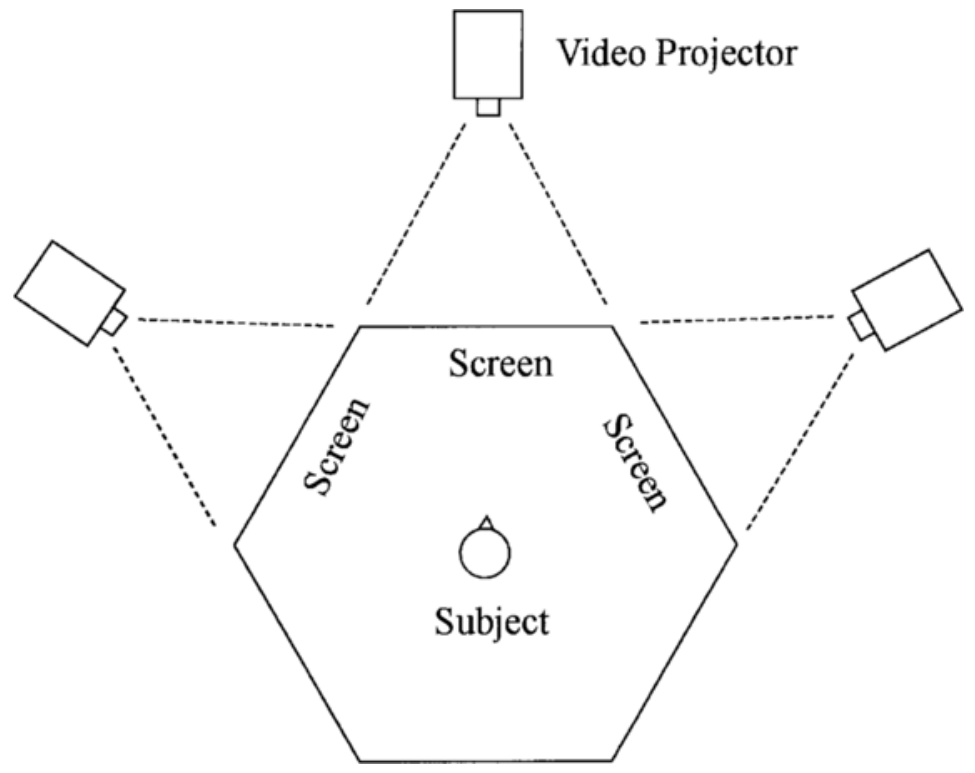

Figure 1. View from above of the screen configuration. The three screens were translucent, and moving displays were projected from behind. The horizontal viewing angle reached $180^{\circ}$, which greatly contributed to the induction of vection. 
play was enough to compel the subjects to feel self-motion. The number of dots on each screen was 512 for all the conditions. The luminance of the dots was $21.3 \mathrm{~cd} / \mathrm{m}^{2}$ at the center of the screen, with a background luminance of $0.3 \mathrm{~cd} / \mathrm{m}^{2}$. The diameter of each dot was about $1 \mathrm{~cm}$. The focus of each projector was made to be soft so that the subjects could not see the RGB dots of the LCD panels. Altering dot positions, synchronized with vertical synch signals at $60 \mathrm{~Hz}$, created motion displays. The dots that disappeared at the edge of each screen reappeared at the opposite edge-that is, the pattern was endless. Since the video signal generated by the computer was distributed to three projectors in parallel, the patterns on the three screens were the same. At the two corners where the screens were connected, dots were not seen, because black screen frames $\left(2-\mathrm{cm}\right.$ width) were occluding them. The corners were $30^{\circ}$ apart in visual angle from the center of the front screen-that is, as seen in peripheral vision. Consequently, the subjects did not notice or mind the discontinuity of dot patterns behind the screen corners caused by a small time lag (from the dot's disappearing at one edge to its reappearing at the opposite edge). In a preliminary experiment, we asked the subjects whether they had noticed the discontinuity and found that they had felt as if the dots moved smoothly across the screens and had not noticed the discontinuity until an experimenter raised this point.

The flow conditions used in the experiments are shown in Figure 2. There were two basic flow patterns: One was an upward or downward dot motion on each screen (linear flow condition), which would induce a sense of self-translation in vertical directions. This pattern was strong enough to cause the subject to perceive himself or herself as descending or ascending. The speed of vertical dot motion was $5.9 \mathrm{deg} / \mathrm{sec}$ in visual angle velocity. The other pattern was a leftward or rightward linear dot motion on each screen (circular flow condition). Although it was not a physically circular movement but an approximation by a half-hexagon, this pattern was enough to make a sufficiently strong impression on the subject's

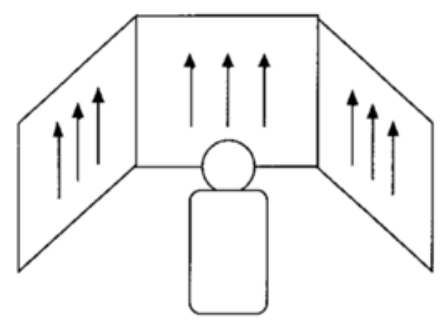

Linear flow condition

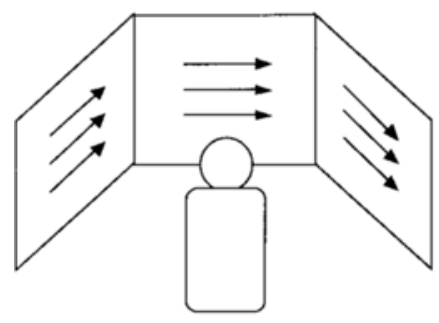

Circular flow condition

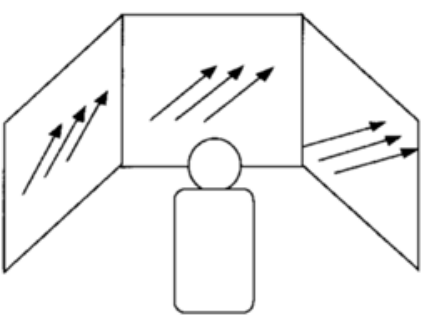

Vector Sum condition

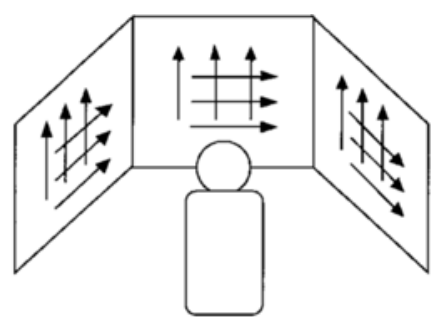

Overlay condition

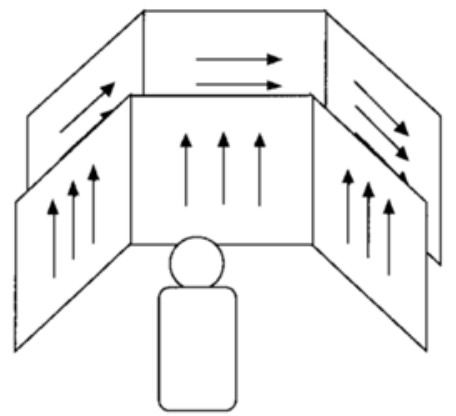

Linear-Near condition

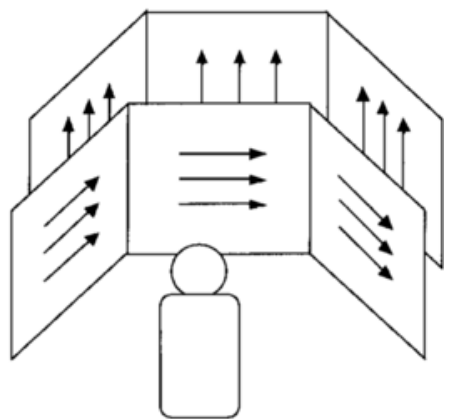

Circular-Near condition

Figure 2. Schematic illustrations of visual stimuli. Samples of flow patterns on three screens are drawn for the linear flow, circular flow, vector sum, and overlay conditions. For the linear-near and circular-near conditions, sample flow patterns on simulated screen configurations are shown. Since the simulated foreground plane was transparent, moving dots were seen simultaneously on the foreground and the background planes. 
perception of self-rotation around a vertical axis. In a preliminary experiment, we found that only two screens connected at right angles, on which linear horizontal dot motion was displayed (i.e., an approximation by a half-square), could induce the perception of self-rotation around a vertical axis. The speed of "circular" dot motion corresponded to $7.8 \mathrm{deg} / \mathrm{sec}$ in visual angle velocity. This speed difference between the linear flow and the circular flow conditions comes from the horizontally longer proportion of the computer pixels and from creating motion displays by shifting dot positions by 1 pixel length per each screen refresh in each direction.

Two compound flow patterns were used. One was the vector sum of the above two flows, consisting of four possible combinations of motion directions - that is, up-left, up-right, down-left, and downright directions on each screen (vector sum condition). Dots moved diagonally across each of the three screens. The other pattern was a simple overlaying of the two, consisting of vertical motions of half of the dots and horizontal motions of the other half, coexisting on each screen (overlay condition). There were also four possible combinations - that is, upward and leftward, upward and rightward, downward and leftward, and downward and rightward motions.

In addition, the two flows were overlaid with phenomenal depth separation under two stereoscopic conditions. This was achieved by presenting stereoscopic images by the anaglyph method. The subjects wore anaglyph glasses consisting of red and blue camera filters and viewed the corresponding colored images through them. Under one condition, the vertical translation of half of the total number of dots was presented on the simulated foreground plane, and circular movement of the other half was presented on the simulated background plane (linear-near condition). The simulated foreground plane was the screen surface itself, and the simulated background plane had a relative disparity of $78 \mathrm{~min}$ of arc in the uncrossed direction. Images on the two side-screens did not present accurate binocular disparities, but the binocularly visible field was almost limited to the front screen when the subjects fixated on the center. There were four possible combinations of vertical translation (upward or downward) and circular movement (leftward or rightward). Under the other stereoscopic condition, the depth order was reversed from the linear-near condition (circular-near condition).

Under the overlay condition, a dot in one flow pattern sometimes intersected another dot in the other flow pattern. In this case, the luminance of the overlapping part was the same as that of the other part of the dots or the other dots. That is, there was neither occlusion nor addition of the luminance. Consequently, the intersection did not provide any depth order information. This was also the case for the stereoscopic conditions. The intersection happened and was displayed in the same way as that noted above for each red and blue color display - that is, for each eye display. When a red dot shared the same spatial position with a blue dot on the screen, both colors were additively displayed - that is, a magenta color. When a magenta dot shared the same spatial position with a blue or red dot, magenta was displayed. Magenta seemed to be red for the right eye and blue for the left eye, just like the other red or blue dots. There was no monocular cue for depth order, such as occlusion, and no addition of luminance for each eye image.

In order to respond to the four kinds of possible compound vection-that is, combinations of perceived self-translation (upward or downward) and self-rotation (leftward or rightward), in addition to simple perceived self-translation or self-rotation- a button that could indicate eight directions was used.

\section{Procedure}

Linear flow, circular flow, vector sum, and overlay conditions. The ideas of vection-that is, induced self-translation and induced self-rotation - were explained to the subjects as they watched flow patterns on the screens. All the subjects clearly understood each description of vection perception. The subject was seated on a chair with his or her head positioned on a chinrest. During each trial, the subject fixated on a circular patch $4.5 \mathrm{~cm}$ in diameter. The patch was placed at the center and $10 \mathrm{~cm}$ in front of the screen directly in front of the subject. After a beeping sound, moving dots appeared on the screens. When the subjects perceived self-ascending or selfdescending motion, they pushed the button to an upper or a lower position from normal, according to the direction of induced selftranslation. When they perceived self-rotation, they pushed the button to the left or to the right, according to the direction of induced self-rotation. When they perceived both, they pushed the button to the middle direction; when they perceived upward self-translation and rightward self-rotation simultaneously, they pushed the button to the up-right direction. When they perceived no vection, they released the button. The output signal of the button was sampled at $60 \mathrm{~Hz}$. The exposure duration for each trial was $120 \mathrm{sec}$, and there were intervals of a few minutes between trials. Trials were randomly performed once through for each of 12 nonstereoscopic conditions (two directions of the linear flow conditions, two directions of the circular flow conditions, four direction combinations for the vector sum conditions, and four direction combinations for the overlay conditions).

Linear-near and circular-near conditions. With right-blue and left-red glasses and images being used, the trials were carried out once for each of the eight conditions (four direction combinations of the linear-near conditions and four direction combinations of the circular-near conditions) in random order. Thereafter, the colors of the glasses and the images on the screens were reversed-that is, right-red and left-blue glasses and images were used-and the trials were performed again. This color change was done in order to ensure that the performance would not be affected by the color difference between the two eye images. In addition, it was determined that all the subjects always reported the correct depth order of the two dot planes. The fixation patch was moved to $1.5 \mathrm{~cm}$ in front of the screen directly facing the subject, in order to make it easier for the subjects to fuse stereo images of both planes. The other procedures used were the same as those under the nonstereoscopic conditions.

\section{RESULTS}

Figures 3 and 4 and Table 1 show the mean duration of each kind of vection (in seconds). $T R+$ means perceived self-translation in the same direction as that of the dot translation, and $T R-$ means perceived self-translation in the opposite direction. $R O+$ means perceived self-rotation in the same direction as that of the dot rotation, and $\mathrm{RO}-$ means perceived self-rotation in the opposite direction. A compound description such as $T R-R O+$ means that $\mathrm{TR}-$ and $\mathrm{RO}+$ occurred simultaneously.

The upper panel in Figure 3 shows the mean durations of the perceived self-translation under the linear flow conditions and of the perceived self-rotation under the circular flow conditions. The results clearly demonstrate that each flow pattern induced vection, in a direction opposite to that of the flow direction, of sufficient strength. The middle panel shows the mean durations of the perceived self-translation and the perceived self-rotation under the vector sum conditions. It is evident that both vections were induced simultaneously in directions opposite to those of the flow components. It is important to note that the perceived compound self-motion was not a linear vection in the middle direction. The subjects actually described feeling an ascending or descending selfmotion, rotating leftward or rightward about a vertical 


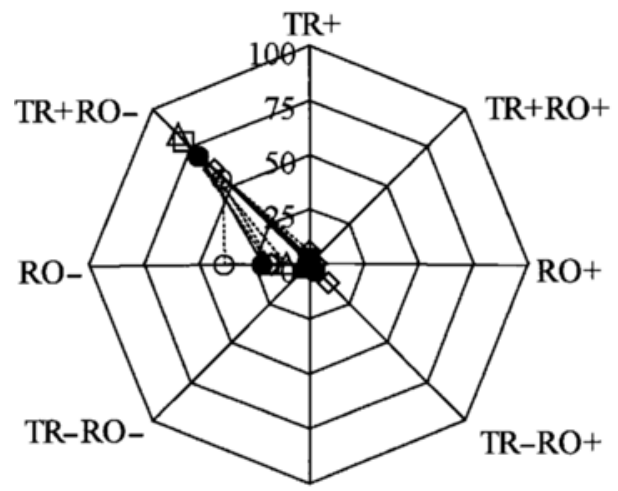

TR-

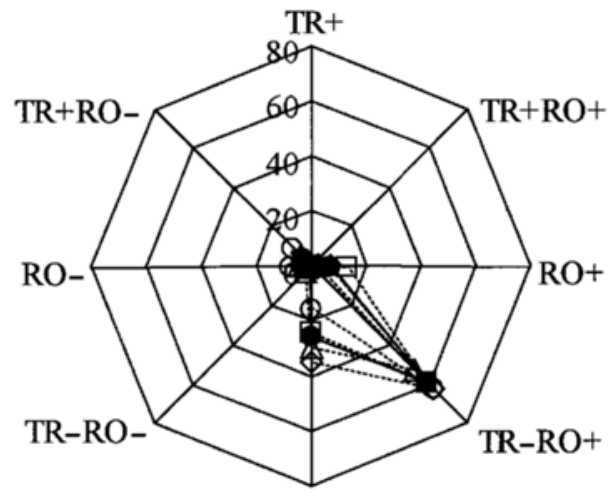

TR-

\section{Linear-Near}

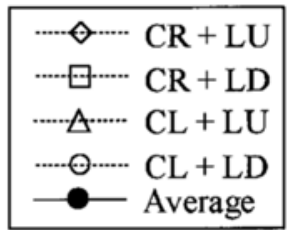

\section{Circular-Near}

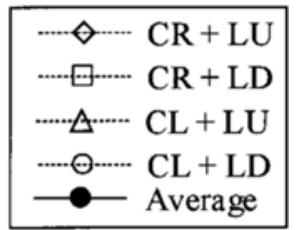

Figure 4. Vection durations in each direction under the linear-near (top panel) and circular-near (bottom panel) conditions. The dominant vection directions are $\mathrm{TR}+\mathrm{RO}-$ and $\mathrm{TR}-\mathrm{RO}+$ for the linear-near and the circularnear conditions, respectively - that is, the direction opposite to that of the stereoscopically farther flow and the same direction as that of the closer flow at the same time. The symbols positioned in the center of each figure indicate zero or near-zero durations in some directions. Precise data values can be found in Table 1. TR+, perceived self-translation in the same direction as that of the dot translation; $T R-$, perceived self-translation in the opposite direction; $\mathrm{RO}+$, perceived self-rotation in the same direction as that of the dot rotation; RO-, perceived self-rotation in the opposite direction.

axis inside a stationary cylindrical structure on which surface light points were attached.

The bottom panel shows the results from experiments in which overlay conditions were used. It demonstrates strong contrasts to the results from the vector sum conditions. When the two flow patterns were simply overlaid, there was a tendency for either vection to occur at any one time. Sometimes a dominant vection reversed spontaneously. Although there were some responses indicating the coexistence of both kinds of vections $(\mathrm{TR}-\mathrm{RO}-$ ), the duration was short, as compared with either vection duration. The results indicate that the two flow patterns causing each kind of vection inhibited each other without producing a phenomenal vector summation (or parallel processing for each vection) when they were simply overlaid.

Quantitatively, there was a large difference between the subjects. For example, for the results obtained from experiments carried out under vector sum conditions, the standard deviations ( $S D \mathrm{~s}$ ) of compound vection duration $(\mathrm{TR}-\mathrm{RO}-$ ) between the subjects were 24.0, 21.9, 38.4, and $20.5 \mathrm{sec}$ for circular-rightward plus linear-upward, circular-rightward plus linear-downward, circularleftward plus linear-upward, and circular-leftward plus linear-downward, respectively. However, under each condition, the response patterns of vection directions were similar in quality and were highly reproducible even when the direction combinations were different. Mean latencies under each condition were varied from 6.0 to $12.6 \mathrm{sec}$. However, no systematic difference between the conditions was found.

Figure 4 shows the mean vection durations under linear-near conditions and circular-near conditions (in seconds). Since the color reversal did not change the performance, the data under both color-filter conditions 
Table 1

Average Vection Durations in Each Category (in Seconds)

\begin{tabular}{|c|c|c|c|c|c|c|c|c|c|}
\hline \multirow[b]{2}{*}{ Flow Type } & \multicolumn{8}{|c|}{ Vection Category } & \multirow[b]{2}{*}{ Total } \\
\hline & $\mathrm{TR}-\mathrm{RO}-$ & $\mathrm{TR}-$ & $\mathrm{RO}-$ & $\mathrm{TR}-\mathrm{RO}+$ & $\mathrm{TR}+\mathrm{RO}-$ & TR+ & $\mathrm{RO}+$ & $\mathrm{TR}+\mathrm{RO}+$ & \\
\hline \multicolumn{10}{|l|}{ Circular flow } \\
\hline Rightward & 0.00 & 0.00 & 81.28 & 0.00 & 0.00 & 0.00 & 7.22 & 0.00 & 88.50 \\
\hline Leftward & 0.00 & 0.00 & 84.52 & 0.00 & 0.00 & 0.00 & 3.41 & 0.00 & 87.93 \\
\hline \multicolumn{10}{|l|}{ Linear flow } \\
\hline Upward & 0.00 & 101.10 & 0.00 & 0.00 & 0.00 & 2.79 & 0.00 & 0.00 & 103.89 \\
\hline Downward & 0.00 & 108.88 & 0.00 & 0.00 & 0.00 & 1.86 & 0.00 & 0.00 & 110.74 \\
\hline \multicolumn{10}{|l|}{ Vector sum } \\
\hline $\mathrm{CR}+\mathrm{LU}$ & 92.58 & 4.62 & 5.28 & 0.08 & 0.00 & 0.05 & 0.26 & 4.28 & 107.16 \\
\hline $\mathrm{CR}+\mathrm{LD}$ & 94.87 & 0.75 & 5.61 & 0.00 & 0.00 & 0.50 & 0.29 & 0.95 & 102.98 \\
\hline $\mathrm{CL}+\mathrm{LU}$ & 70.79 & 7.43 & 23.36 & 0.00 & 0.00 & 0.03 & 1.17 & 1.68 & 104.46 \\
\hline $\mathrm{CL}+\mathrm{LD}$ & 100.36 & 1.29 & 3.61 & 0.02 & 0.28 & 0.00 & 0.16 & 1.13 & 106.85 \\
\hline Average & 89.65 & 3.52 & 9.47 & 0.02 & 0.07 & 0.15 & 0.47 & 2.01 & 105.36 \\
\hline \multicolumn{10}{|l|}{ Overlay } \\
\hline $\mathrm{CR}+\mathrm{LU}$ & 8.19 & 33.68 & 25.06 & 10.78 & 16.71 & 1.88 & 2.56 & 0.09 & 98.95 \\
\hline $\mathrm{CR}+\mathrm{LD}$ & 18.57 & 47.75 & 22.51 & 6.05 & 4.95 & 0.16 & 1.06 & 0.05 & 101.11 \\
\hline $\mathrm{CL}+\mathrm{LU}$ & 12.59 & 31.32 & 34.86 & 12.63 & 7.67 & 1.45 & 1.34 & 0.10 & 101.98 \\
\hline $\mathrm{CL}+\mathrm{LD}$ & 9.15 & 36.92 & 44.78 & 2.79 & 7.58 & 0.28 & 0.80 & 0.00 & 102.30 \\
\hline Average & 12.12 & 37.42 & 31.80 & 8.06 & 9.23 & 0.95 & 1.44 & 0.06 & 101.09 \\
\hline \multicolumn{10}{|l|}{ Linear-near } \\
\hline $\mathrm{CR}+\mathrm{LU}$ & 0.16 & 1.07 & 17.39 & 12.53 & 61.09 & 5.62 & 3.14 & 0.00 & 100.99 \\
\hline $\mathrm{CR}+\mathrm{LD}$ & 0.04 & 0.98 & 18.34 & 2.29 & 79.71 & 2.78 & 0.20 & 0.01 & 104.36 \\
\hline $\mathrm{CL}+\mathrm{LU}$ & 2.08 & 0.25 & 10.96 & 2.06 & 84.62 & 1.83 & 0.13 & 0.00 & 101.93 \\
\hline $\mathrm{CL}+\mathrm{LD}$ & 0.95 & 0.52 & 38.55 & 0.00 & 56.04 & 2.16 & 0.02 & 0.02 & 98.26 \\
\hline Average & 0.81 & 0.71 & 21.31 & 4.22 & 70.37 & 3.10 & 0.87 & 0.01 & 101.38 \\
\hline \multicolumn{10}{|l|}{ Circular-near } \\
\hline $\mathrm{CR}+\mathrm{LU}$ & 0.00 & 34.33 & 0.17 & 63.19 & 0.00 & 0.18 & 7.00 & 0.00 & 104.86 \\
\hline $\mathrm{CR}+\mathrm{LD}$ & 1.35 & 24.17 & 0.36 & 59.84 & 1.35 & 0.40 & 13.16 & 0.00 & 100.65 \\
\hline $\mathrm{CL}+\mathrm{LU}$ & 3.02 & 29.08 & 4.29 & 53.75 & 5.66 & 0.07 & 2.15 & 0.00 & 98.03 \\
\hline $\mathrm{CL}+\mathrm{LD}$ & 0.75 & 15.08 & 7.79 & 63.28 & 10.03 & 0.02 & 6.30 & 0.00 & 103.25 \\
\hline Average & 1.28 & 25.67 & 3.15 & 60.01 & 4.26 & 0.17 & 7.15 & 0.00 & 101.70 \\
\hline
\end{tabular}

Note-TR+, perceived self-translation in same direction as dot translation; TR -, perceived self-translation in opposite direction; $\mathrm{RO}+$, perceived self-rotation in same direction as dot rotation; $\mathrm{RO}-$, perceived self-rotation in opposite direction; CR, circular-rightward; CL, circular-leftward; LU, linear-upward; LD, linear-downward.

were averaged - that is, each symbol in the figure represents the mean of 20 trials. The response patterns of the four direction combinations were the same under each condition. Self-translation and self-rotation were induced simultaneously when the two flow patterns were overlaid with stereoscopic depth separation. Under linearnear conditions, mainly self-translation in the same direction and self-rotation in the opposite direction to that of the dot motion $(\mathrm{TR}+\mathrm{RO}-$ ) were perceived. The subjects described feeling a self-rotation around a vertical axis inside a stationary cylindrical structure and, at the same time, a self-ascending (or self-descending) according to the foreground motion of the light points, which were perceived to ascend (or descend) faster than the subject's perceived self-ascending (or self-descending). On the other hand, under circular-near conditions, mainly selftranslation in the opposite direction and self-rotation in the same direction to that of the dot motion $(\mathrm{TR}-\mathrm{RO}+)$ were perceived. These results clearly indicate that when two flows move at phenomenally different depth planes, the background flow induces vection in the direction opposite to the flow direction and the foreground flow induces vection in the same direction as the flow direction. There were also some $\mathrm{RO}$ - responses under linear-near conditions and TR - responses under circular-near conditions - that is, the directions opposite to those of the background flows. This shows the strength of the background flows for the induction of vection, as compared with foreground flows.

Figure 5 provides a summary of the results. The upper panel shows the total vection durations in each direction, averaged within each flow combination condition-that is, the vector sum, overlay, linear-near, or circular-near conditions. Each symbol indicates the mean durations of 44 trials under vector sum or overlay conditions and of 80 trials under linear-near or circular-near conditions (in seconds). The lower panel shows the normalized response patterns. That is, in each flow combination condition, the longest vection duration among the eight response categories was assigned " 1 " and then the proportions of durations in the other categories to the longest one were calculated. The differences in the response patterns among the conditions noted above are illustrated more clearly. These figures demonstrate that the TR-ROpercept is dominant under the vector sum condition, that $\mathrm{TR}-$ and $\mathrm{RO}-$ are dominant under the overlay condition, that $\mathrm{TR}+\mathrm{RO}-$ is dominant under the linear-near condition, and that $\mathrm{TR}-\mathrm{RO}+$ is dominant under the circular-near condition.

We also conducted statistical analyses for the data. However, it was difficult to directly analyze the differences among the response patterns consisting of the cat- 


\section{Vection Duration $(\mathrm{sec})$}

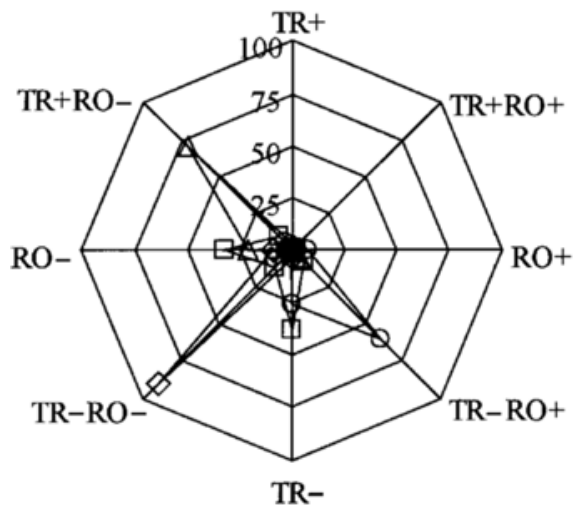

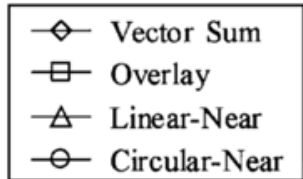

\section{Proportion}

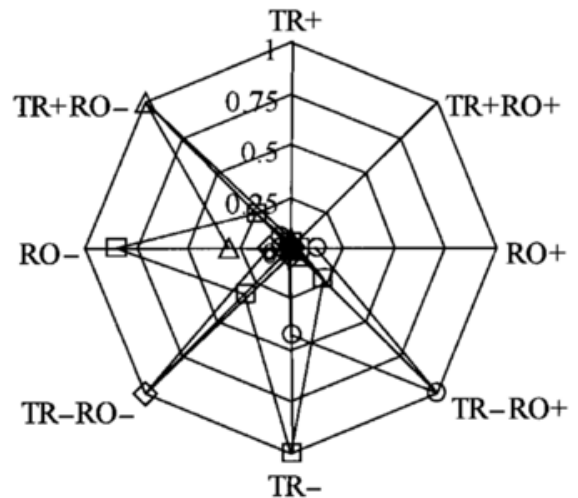

$$
\begin{aligned}
& \diamond-\text { Vector Sum } \\
& \square \text { Overlay } \\
& \triangle \text { Linear-Near } \\
& \oslash \text { Circular-Near }
\end{aligned}
$$

Figure 5. Average vection durations in each direction under vector sum, overlay, linear-near, and circular-near conditions. The upper panel shows the durations in seconds, and the lower panel shows them in normalized values that are proportional to the longest duration among the durations in eight categories for each flow combination condition. The symbols positioned in the center of each figure indicate zero or near-zero durations in some directions. TR + , perceived self-translation in the same direction as that of the dot translation; TR-, perceived self-translation in the opposite direction; $R O+$, perceived selfrotation in the same direction as that of the dot rotation; $\mathrm{RO}-$, perceived selfrotation in the opposite direction.

egorical response data. Therefore, before conducting a statistical analysis, we converted the response patterns to vection scores, which represent a qualitative difference between the response patterns. We assigned "1" for $\mathrm{RO}$ - or TR - responses (i.e., ordinary vection responses), whereas " -1 " was assigned for $\mathrm{RO}+$ or $\mathrm{TR}+$ responses (i.e., opposite vection responses). Accordingly, TR-ROresponses were assigned " 2 ," whereas $\mathrm{TR}-\mathrm{RO}+$ or $\mathrm{TR}+\mathrm{RO}-$ responses were assigned " 0 ." $\mathrm{TR}+\mathrm{RO}+$ responses were assigned " -2. ."

When the total vection duration acquired from one trial consists of $\mathrm{TR}-\mathrm{RO}-$ responses only, the score of the trial is " 2 ," whereas if there are TR - or RO- responses only, the score is " 1. ." When $50 \%(25 \%)$ of the total vection duration from a trial consists of TR $-\mathrm{RO}-$ responses and the other $50 \%$ ( $75 \%$ ) consists of $\mathrm{RO}-$ responses, the score of the trial is $1.5(1.25)$. That is, the vection score of a trial is the averaged vection score through the trial that may include a few categories of responses. The score should vary within a range from -2 to 2 .

Figure 6 shows the vection scores. For the vector sum conditions, the score under each flow-direction combination condition, averaged over 11 subjects, ranged from 1.61 to 1.90 , meaning that $\mathrm{TR}-\mathrm{RO}-$ was the dominant perception. Under the overlay conditions, the scores ranged from 0.71 to 1.05 , meaning that $\mathrm{TR}-$ or $\mathrm{RO}-$ was the dominant perception, including some $\mathrm{TR}-\mathrm{RO}-$, $\mathrm{TR}-\mathrm{RO}+$, and $\mathrm{TR}+\mathrm{RO}-$ perceptions (see Table 1). Under the linear-near or the circular-near condition, the scores averaged over 10 subjects ranged from 0.19 to 0.33 or from 0.28 to 0.45 , respectively, meaning that $\mathrm{TR}+\mathrm{RO}-$ or $\mathrm{TR}-\mathrm{RO}+$ was the dominant perception, including 


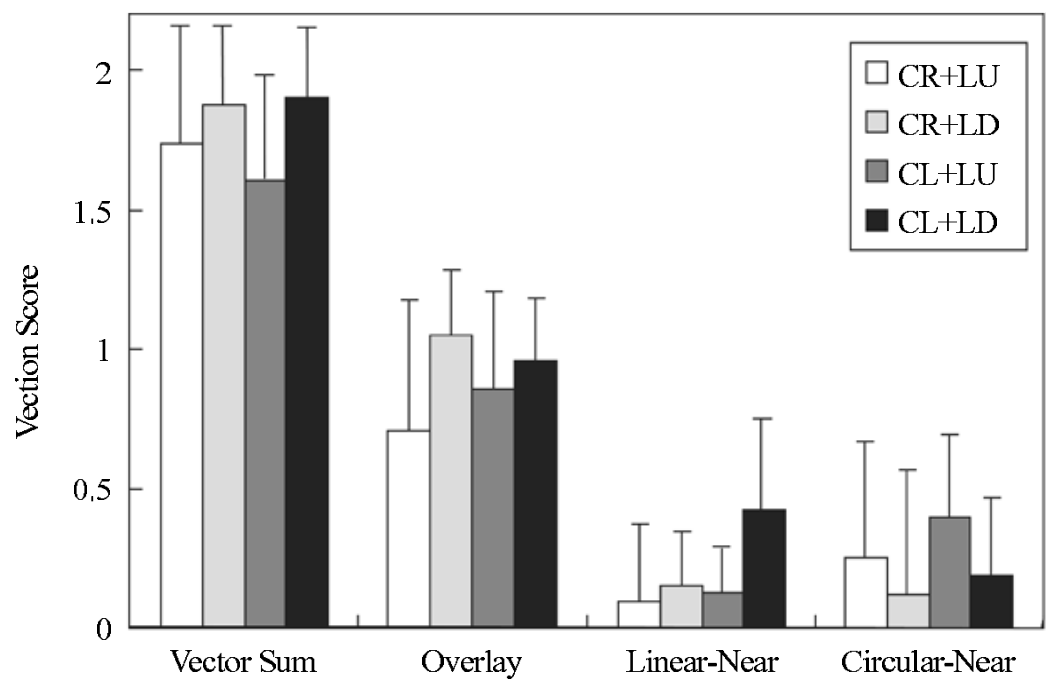

Figure 6. Average vection scores. Vertical lines in the figure indicate standard deviations. See the text for a detailed description. CR, circular-rightward; CL, circularleftward; LU, linear-upward; LD, linear-downward.

some $\mathrm{RO}-$ or $\mathrm{TR}-$ perceptions, respectively. These scores represent the differences among the response patterns under those conditions well.

We first conducted a three-way analysis of variance (ANOVA) for the averaged scores. The three factors were vertical flow directions (upward or downward), circular flow directions (leftward or rightward), and flow combination types (vector sum, overlay, linear-near, and circular-near). There was a significant main effect of the flow combination types $[F(3,3)=189.89, p<.001]$. The least significant difference (LSD) tests revealed that there were significant differences between all the pairs of flow combination types $(p<.01)$, except for a pair of linear-near and circular-near conditions $(p>.05)$. Although there was no significant difference between the linear-near and the circular-near conditions, note that there was a difference in the dominant vection categories-that is, $\mathrm{TR}+\mathrm{RO}-$ under the linear-near condition or $\mathrm{TR}-\mathrm{RO}+$ under the circular-near condition was dominant, as is shown in Figure 5 and Table 1. In both cases, it is common that the background flow induces ordinary vection and the foreground flow induces an opposite vection at the same time. The other main effects and their interactions were not significant $(p>.05)$.

Next, we again conducted a three-way repeated measures ANOVA for the scores from the 4 subjects who carried out trials under all of the conditions. There was a significant main effect of the flow combination types $[F(3,48)=136.74, p<.0001]$. The LSD tests revealed that there were significant differences between all the pairs of flow combination type conditions $(p<.0001)$, except for a pair of linear-near and circular-near conditions $(p>.05)$. The other main effects and their interactions were not significant $(p>.05)$. This result replicates the first one. These analyses give strong assurance that the response patterns across the eight categories are different among the flow combination type conditions.

Figure 7 shows the average sustained durations, which are the total vection durations divided by the frequency of vection occurrence for each response category. The sustained durations may reflect the relative stability of each vection percept. Generally, a response category with longer sustained durations corresponded to that with longer total durations (see Figure 5). However, under the overlay conditions, there were not large differences among the sustained durations for TR $-(8.21 \mathrm{sec})$, RO $-(7.81 \mathrm{sec}), \mathrm{TR}-\mathrm{RO}-(6.32 \mathrm{sec})$, and TR+RO$(6.51 \mathrm{sec})$ percepts, whereas, as is shown in Table 1, the total durations for $\mathrm{TR}-(37.42 \mathrm{sec})$ and $\mathrm{RO}-(31.80 \mathrm{sec})$ percepts were more than twice as long as the total duration for the TR-RO- $(12.12 \mathrm{sec})$ or the TR+RO$(9.23 \mathrm{sec})$ percept. This indicates that the relative stability was not so different among the four percepts and that the $\mathrm{TR}-\mathrm{RO}-$ or $\mathrm{TR}+\mathrm{RO}-$ percept occurred less frequently. In fact, the frequency of each vection occurrence, averaged over 44 trials, under this condition was 4.59 (per trial) for $\mathrm{TR}-$ or 4.18 for the $\mathrm{RO}$ - percept, whereas it was 2.09 for $\mathrm{TR}-\mathrm{RO}-$ or 1.45 for the $\mathrm{TR}+\mathrm{RO}-$ percept.

The sustained duration data also illustrate that, under the overlay conditions, each percept lasted a shorter range of time, as compared with the $\mathrm{TR}-\mathrm{RO}-$ percept under the vector sum conditions $(27.04 \mathrm{sec})$, TR $-\mathrm{RO}+$ under the circular-near conditions $(23.54 \mathrm{sec})$, and $\mathrm{TR}+\mathrm{RO}-$ under the linear-near conditions $(20.82 \mathrm{sec})$. These shorter sustained durations do not mean that the effectiveness of the flows for inducing vection was totally weak under the overlay condition, because the total durations - that is, the sums of vection durations in all the categories-were not shorter than those under the 


\section{Vector Sum}

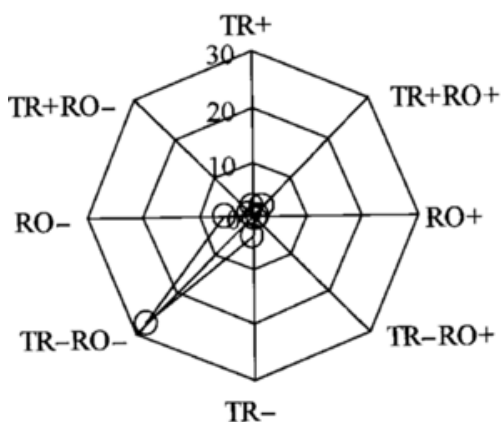

Overlay

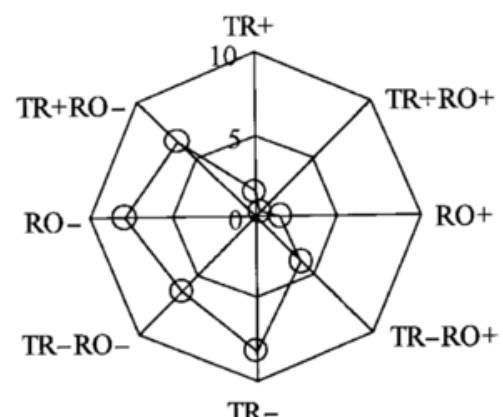

Linear-Near

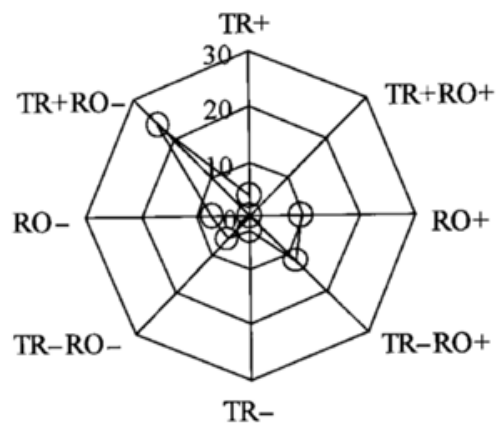

Circular-Near

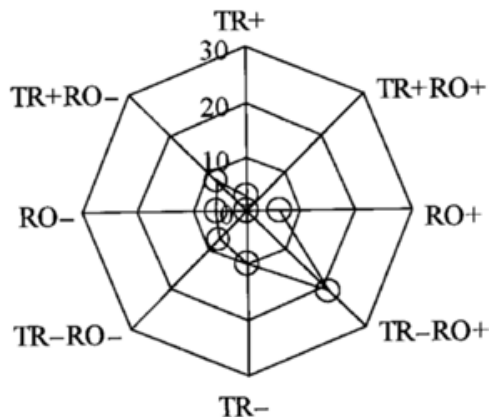

Figure 7. Average sustained durations in each direction under vector sum, overlay, linear-near, and circular-near conditions (in seconds). These are the total durations in each direction divided by the frequency of vection occurrence in each direction. TR+, perceived self-translation in the same direction as that of the dot translation; TR-, perceived self-translation in the opposite direction; $\mathrm{RO}+$, perceived self-rotation in the same direction as that of the dot rotation; $\mathrm{RO}^{-}$, perceived self-rotation in the opposite direction.

other flow combination type conditions, as will be noted below.

The upper panel in Figure 8 shows the average total duration in a trial. These values are the sums of the vection durations in the eight response categories within a trial and are shown in Table 1. No significant difference among the conditions was found with a three-way ANOVA $(p>.05)$. The middle panel in Figure 8 shows the sums of the frequencies of vection occurrence in the eight categories within a trial. As is clearly shown in the figure, the values are larger under the overlay condition than under the other flow combination type conditions. A three-way ANOVA revealed that the main effect of flow combination types (vector sum, overlay, linear-near, and circular-near conditions) was significant $[F(3,3)=46.90$, $p<.01]$. The LSD tests revealed that the differences between the overlay condition and each of the other flow combination type conditions were significant $(p<.01)$ and that the differences between the other pairs of flow combination type conditions were not significant $(p>$ .05 ). The main effects of vertical flow directions (upward or downward) and circular flow directions (leftward or rightward) were not significant $(p>.05)$. No interaction was significant $(p>.05)$. These statistics give assurance that, under the overlay conditions, the subjects changed vection response more frequently than under other flow combination type conditions. The lower panel in Figure 8 shows the averaged sustained durations that were calculated with the total durations (the upper panel) divided by the summed frequencies of vection occurrence in the eight categories (the middle panel). As would be expected from the above results, the sustained durations under the overlay conditions were shorter than those under the other conditions. A three-way ANOVA revealed that the main effect of flow combination types (vector sum, overlay, linear-near, and circular-near conditions) was significant $[F(3,3)=18.65, p<.05]$. The LSD tests revealed that the difference between the overlay and the vector sum conditions was significant $(p<.01)$. The differences between the overlay and the linear-near conditions and between the overlay and the circular-near conditions were also significant $(p<.05)$. The main effects of the vertical flow directions (upward or downward) and the circular flow directions (leftward or rightward) were not significant $(p>.05)$. No interaction was significant $(p>.05)$. These simple statistics evidently 

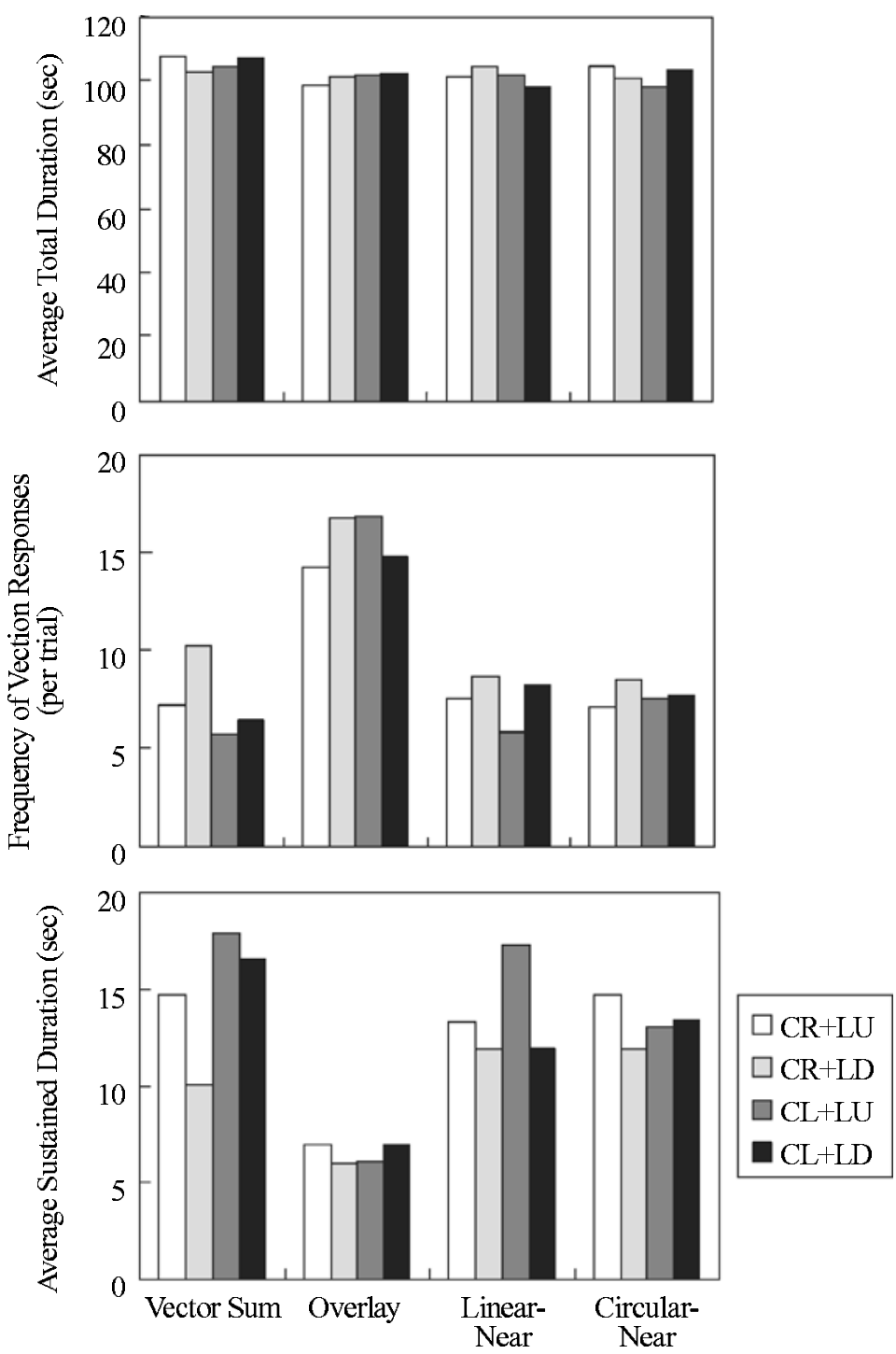

Figure 8. Average total vection durations, average total frequencies of vection responses, and average sustained durations in a trial. The average sustained durations are calculated by the average total durations divided by the average total frequencies of vection responses. $\mathrm{CR}$, circular-rightward; CL, circularleftward; LU, linear-upward; LD, linear-downward.

indicate that vection was unstable and that the direction was easy to change under the overlay condition, as compared with that under the vector sum, linear-near, or circular-near conditions.

To summarize the results, each of the linear and circular flows induced each vection, a sense of translational or rotational self-motion, in the opposite direction to the flow when they were presented alone. When the motion vectors were summed in the display, the subjects tended to perceive compound self-motion in a direction opposite to both flows. However, when they were simply overlaid on the screens, the subjects tended to perceive either a linear or a circular vection, and vection direction cate- gories frequently changed. On the other hand, when they were overlaid with binocular disparity, the subjects tended to perceive a compound self-motion in a different way from that under the vector sum conditions. That is, under the linear-near conditions, they felt a rotational self-motion in a direction opposite to the background circular flow direction, and at the same time, they felt a translational self-motion in the same direction as the foreground linear flow direction. Under circular-near conditions, they felt a translational self-motion in a direction opposite to the background linear flow direction, and at the same time, they felt a rotational self-motion in the same direction as the foreground circular flow direction. 


\section{DISCUSSION}

In the present paper, we have demonstrated with two procedures the coexistence of two kinds of vection - that is, induced self-translation and induced self-rotation. This coexistence occurred when the vector sum of both linear and circular flows was presented or when the two flows were overlaid with stereoscopic depth separation. Without depth separation, the tendency was for either vection to occur. This is the first report that has shown the coexistence of two different kinds of vection-that is, vertical linear vection and circular vection around a vertical axis. In some optical flow experiments, two different types of flows were superimposed (Duffy \& Wurtz, 1993; Grigo \& Lappe, 1998). However, no such experiment has been done in the vection paradigm, and no research has measured compound vection.

$\mathrm{TR}-\mathrm{RO}-$ perception arose mainly under the vector sum conditions. This result could suggest that two kinds of global flow detectors work in parallel to induce vection. Physiologically, neurons that selectively respond to specific flow patterns (e.g., expansion-contraction or rotation), having large receptive fields, have been identified in the medial superior temporal area (Tanaka, Fukada, \& Saito, 1989; Tanaka \& Saito, 1989). On the other hand, if both of the two flows under the linear-near or the circularnear conditions were detected in parallel to produce vection sensations, the results would be the same as those under the vector sum conditions. However, evidently, this is not the case. Although both flows affected vection, the direction was inconsistent with those under the vector sum conditions. That is, vection under stereoscopic conditions was perceived in the direction opposite to the background flow and in the same direction as the foreground flow ( $\mathrm{TR}+\mathrm{RO}-$ under the linear-near conditions or $\mathrm{TR}-\mathrm{RO}+$ under the circular-near conditions). This difference indicates that there is a difference in the process of inducing compound vection between the vector sum condition and both stereoscopic (linearnear and circular-near) conditions. As was noted earlier, some researchers have reported the foreground flow effect (Howard \& Heckmann, 1989; Nakamura \& Shimojo, 2000), which produces vection in the same direction as the foreground motion. It is thought that the foreground flow effect and vection determined by the background flow occurred simultaneously under stereoscopic conditions, resulting in coexistence of two kinds of vections.

The difference between the overlay condition and the two stereoscopic conditions (linear-near and circularnear conditions) was the existence of stereoscopic depth separation. However, the reported vection was quite different. Under the overlay conditions, it was difficult for both vections to coexist. It is thought that there arose apparent reversals of a near-far relationship between the two flows and that only the apparently far flow induced vection, whereas the apparently near flow was ignored, as Ohmi et al. (1987) suggested. According to the sub- ject's verbal reports, such apparent reversals actually happened. However, the foreground flow effect as seen under the stereoscopic conditions was not seen under the overlay conditions. Stereoscopic depth separation seems necessary for the foreground flow effect-that is, surface segregation seems insufficient for the effect. Quantitative research on the amount of necessary depth and on which depth cue is effective should be done in the future.

Howard and Heckmann (1989) explained the foreground flow effect as follows: Motion of the dots on the background plane was induced by motion on the foreground plane. Then the induced motion of the background induced vection. They called this phenomenon contrastmotion vection (Howard \& Heckmann, 1989) or inducedmotion vection (Howard, 1990). The contrast-motion vection explanation assumes that the number of flows mediating the sensation of vection should be limited to one-that is, the farthest. According to this idea, our results may be explained as follows: The simulated background flow mediates a relative motion component to the simulated foreground flow as induced motion, in addition to the original motion component. Then flow detectors for self-translation and self-rotation may respond in parallel to both flow components within the virtually vectorsummed background flow, as was observed under the vector sum conditions, although the resultant direction is different.

There is a common characteristic found in both induced motion and the foreground flow effect found here that supports the contrast-motion vection hypothesis. In preliminary observations, we tested moving foreground plus stationary background conditions. In this case, vection was hardly perceived, as was noted by Ohmi et al. (1987) and Nakamura and Shimojo (1999). That is, the effect of foreground motion was weak, if there was any, when a background plane was stationary. On the other hand, our present results, together with those of Nakamura and Shimojo (2000), indicate a rather strong effect in conditions under which the background plane moves orthogonally to the foreground-plane motion. Induced motion of an object, observed within a horizontally moving frame, is much clearer when the object inside the frame moves vertically (resulting in perceived diagonal motion of the object) than when it is stationary. The hypothesis for the foreground flow effect, based on induced motion, can explain why the foreground flow effect is stronger when the background plane moves orthogonally.

On the other hand, evidence against the contrast-motion vection hypothesis also exists. Induced motion should be strongest when an inducer and a target are in the same plane (Gogel \& MacCracken, 1979). However, when two flows are overlaid without binocular disparity (under the overlay conditions), the results show that either form of vection occurs - that is, no contrast-motion vection. Nakamura and Shimojo (1999) suggested the possibility of direct contribution of the foreground flow, showing that reported vection strength cannot be explained by the relative motion component between foreground and back- 
ground motion. Recently, Nakamura and Shimojo (2000) showed that the visual stimulus composed of a vertically moving random-dot foreground and a vertically striped background, where motion induction on the background was impossible, can induce inverted vection. They proposed that misregistration of eye movement information due to a suppression of optokinetic nystagmus caused vection in the same direction as the foreground flow, whereas the background flow induced normal vection. This assumption may require further studies. There has been little research done in which this phenomenon has been investigated.

We should also consider the role of attention, especially for the results under the overlay conditions. Recent research has revealed an effect of attentional modulations on the detection of motion or on motion aftereffects when two components of motion overlap (e.g., Lankheet $\&$ Verstraten, 1995). Kitazaki and Sato (2003) measured the effect of voluntary attention on vection direction with a display including upward and downward optical motion. Their results showed that vection arose mainly in the direction opposite to the nonattended flow. In our display under the overlay conditions, attentional shift from one flow to the other, caused by fatigue or adaptation of the motion-processing system, may have changed flow dominance for vection. However, it is also possible that apparent reversals of the figure-ground relationship and the depth order of the two flows may have changed the vection direction and caused attentional shift. In the heading paradigm, Wann, Swapp, and Rushton (2000) demonstrated the effect of attention on the headingdirection judgments. They showed that recovering heading when a radial flow is contaminated with a rotational component arising from viewpoint rotation required focused attention. This might be indirect evidence showing that attention has an effect on the direction of vection induced from compound optical flows. However, under stereoscopic conditions in which the depth order of the two flows was explicitly indicated, reversals of vection direction were rare. Even if attentional shift or attentional tracking on either flow played an important role in the changes of vection direction under the overlay conditions, the effect seems to arise mainly when two flows are on the same disparity plane (or when no disparity cue is available).

One could argue that the eyes may have tracked one of the two flows and that vection occurred in the same direction as the tracking. Since we used a fixation patch with a diameter of $4.3^{\circ}$ of visual angle, eye movements could occur within this small area. If an eye tracked leftward or rightward rotation within this area, possible tracking duration would be $0.55 \mathrm{sec}$ at most, because the rotational flow speed was $7.8 \mathrm{deg} / \mathrm{sec}$. This may suggest that if there was an effect of eye movement information on vection in our stimuli, it would not be from continuous and smooth eye tracking, but from the misregistered information about eye movement noted above.

Finally, one important series of reports, beginning with Duffy and Wurtz (1993), should be referred to. They overlaid expanding motion and horizontally translational motion on the same plane. There arose an illusory shift of the focus of expansion in the direction of element translation. This illusion was explained by a compensation process for translational flow caused by eye movements (Duffy \& Wurtz, 1993), the induced motion mechanism (Meese, Smith, \& Harris, 1995), or both (Pack \& Mingolla, 1998). Grigo and Lappe (1998) associated the illusion with a self-motion situation that they called egomotion scenario. They overlaid the two flows with binocular disparity and found that when expansion was in front, the illusory shift was larger than that arising when translation was in front. They asserted that the whole flow pattern was consistent with that arising during forward self-translation with eye rotation. The direction of the shifted focus of expansion was assumed to be the heading direction in their ego-motion scenario (Grigo \& Lappe, 1998; Lappe \& Rauschecker, 1995).

In a sense, this scenario is consistent with Nakamura and Shimojo's (2000) explanation for their inverted vection. That is, one of the two flows overlaid with disparity is related to body translation information, and the other is related to eye movement information. Although Nakamura and Shimojo (2000) assumed that the background flow is related to body translation and the foreground flow is related to eye movement, Grigo and Lappe (1998) assumed the opposite. However, it is possible that each effect is a specific phenomenon related to each combination of flow patterns. For example, in the display of Grigo and Lappe, the visual system cannot assume that the expanding flow in any depth plane represents eye movement information, whereas it is possible to interpret either flow as representing eye movement in the displays of Nakamura and Shimojo (2000) and in ours. We are planning other vection experiments, using various flow patterns overlaid with and without phenomenal depth, to solve the problem. These studies will suggest how vection and heading paradigms can be combined, which is an area that has not received much attention in the literature.

\section{REFERENCES}

Andersen, G. J., \& Braunstein, M. L. (1985). Induced self-motion in central vision. Journal of Experimental Psychology: Human Perception \& Performance, 11, 122-132.

Brandt, T., Dichgans, J., \& Koenig, E. (1973). Differential effects of central versus peripheral vision on egocentric and exocentric motion perception. Experimental Brain Research, 16, 476-491.

Duffy, C. J., \& Wurtz, R. H. (1993). An illusory transformation of optical flow fields. Vision Research, 33, 1481-1490.

Gogel, W. C., \& MacCracken, P. J. (1979). Depth adjacency and induced motion. Perceptual \& Motor Skills, 48, 343-350.

Grigo, A., \& LAPPE, M. (1998). Interaction of stereo vision and optic flow processing revealed by an illusory stimulus. Vision Research, 38, 281-290.

HowARD, I. P. (1990). Spatial vision within egocentric and exocentric frames of reference. In S. R. Ellis (Ed.), Pictorial communication in virtual and real environments (pp. 338-358). London: Taylor \& Francis.

Howard, I. P., Cheung, B., \& LANDOLt, J. (1988). Influence of vection axis and body posture on visually-induced self rotation. Advisory Group for Aerospace Research \& Development, 433, 15-1 to 15-8. Howard, I. P., \& Heckmann, T. (1989). Circular vection as a function 
of the relative sizes, distances and positions of two competing visual displays. Perception, 18, 657-667.

Iто, H., \& ShiвaO, T. (1999). Motion direction distribution as a determinant of circular vection. Perceptual \& Motor Skills, 89, 564-570.

Johansson, G. (1977). Studies on visual perception of locomotion.Perception, 6, 365-376.

KANO, C. (1991). The perception of self-motion induced by peripheral visual information in sitting and supine postures. Ecological Psychology, 3, 241-252.

KitazaKi, M., \& SATo, T. (2003). Attentional modulation of self-motion perception. Perception, 32, 475-484.

Lankheet, M. J., \& Verstraten, F. A. J. (1995). Attentional modulation of adaptation to two-component transparent motion. Vision Research, 35, 1401-1412.

LAPPE, M., \& RAUSCHECKER, J. P. (1995). An illusory transformation in a model of optical flow processing. Vision Research, 35, 1619-1631.

Meese, T. S., Smith, V., \& Harris, M. G. (1995). Induced motion may account for the illusory transformation of optic flow fields found by Duffy \& Wurtz. Vision Research, 35, 981-984.

NaKamura, S., \& Shimojo, S. (1998). Stimulus size and eccentricity in visually induced perception of horizontally translational self-motion. Perceptual \& Motor Skills, 87, 659-663.

Nakamura, S., \& Shimojo, S. (1999). Critical role of foreground stimuli in perceiving visually induced self-motion (vection). Perception, 28, 893-902.

NAKAmura, S., \& Shimojo, S. (2000). A slowly moving foreground can capture an observer's self-motion: A report of a new motion illusion. Inverted vection. Vision Research, 40, 2915-2923.
OHмi, M., \& Howard, I. P. (1988). Effect of stationary objects on illusory forward self-motion induced by a looming display. Perception, 17, 5-12.

Ohmi, M., Howard, I. P., \& Landolt, J. P. (1987). Circular vection as a function of foreground-background relationships. Perception, 16, 17-22.

Pack, C., \& Mingolla, E. (1998). Global induced motion and visual stability in an optic flow illusion. Vision Research, 38, 3083-3093.

Palmisano, S. (1996). Perceiving self-motion in depth: The role of stereoscopic motion and changing-size cues. Perception \& Psychophysics, 58, 1168-1176.

Palmisano, S., Gillam, B. J., \& Blackburn, S. G. (2000). Globalperspective jitter improves vection in central vision. Perception, 29, 57-67.

Tanaka, K., Fukada, Y., \& Saito, H. (1989). Underlying mechanisms of the response specificity of expansion/contraction and rotation cells in the dorsal part of the medial superior temporal area of the macaque monkey. Journal of Neurophysiology, 62, 642-656.

TANAKA, K., \& SAITo, H. (1989). Analysis of motion of the visual field by direction, expansion/contraction, and rotation cells clustered in the dorsal part of the medial superior temporal area of the macaque monkey. Journal of Neurophysiology, 62, 626-641.

WANN, J. P., SwaPp, D., \& Rushton, S. K. (2000). Heading perception and the allocation of attention. Vision Research, 40, 2533-2543.

(Manuscript received November 30, 1999; revision accepted for publication February 18, 2003.) 